Artículo

\title{
Competitividad y rentabilidad de la producción de frutillas en Jalisco
}

\begin{abstract}
Edgar Ricardo Lagunes-Fortiz ${ }^{1}$
Erika Lagunes-Fortiz ${ }^{3 \S}$

Alma Alicia Gómez-Gómez ${ }^{1}$

Juan Antonio Leos-Rodríguez ${ }^{2}$

José Miguel Omaña-Silvestre ${ }^{4}$

${ }^{1}$ División de Ciencias Económico Administrativas-Universidad Autónoma Chapingo. Carretera MéxicoTexcoco km 38.5. Chapingo, Estado de México, México. CP. 56230. (ricardo_lafo@hotmail.com; almaaliciamx@yahoo.com). ${ }^{2}$ Centro de Investigaciones Económicas, Sociales y Tecnológicas de la Agroindustria y la Agricultura Mundial-Universidad Autónoma Chapingo. Carretera México-Texcoco km 38.5, Chapingo, Estado de México. CP. 56230. Tel. 5959521000 , ext. 6019 y 6020 (jleos@ ciestaam.edu.mx). ${ }^{3}$ Departamento de Fitotecnia-Universidad Autónoma Chapingo. Carretera México-Texcoco km 38.5. Chapingo, Texcoco, Estado de México. CP. 56230. Tel. 595 9521500, ext. 6133. ${ }^{4}$ Posgrado Socioeconomía, Estadística e Informática-Economía-Colegio de Postgraduados. Montecillo, Texcoco, Estado de México. CP. 56230. (miguelom@colpos.mx).
\end{abstract}

${ }^{\S}$ Autora para correspondencia: erika_lagunes@ hotmail.com.

\section{Resumen}

Durante 2017, Jalisco fue el segundo estado con mayor aportación al PIB agrícola nacional (SIAP, 2020). El cultivo de berries es una importante actividad socioeconómica de este estado, los cuales incluyen el arándano (Vaccinium spp.), frambuesa (Rubus idaeus) y zarzamora (Rubus ulmifolius). Jalisco es el principal productor de frambuesa, el segundo de zarzamora, y el cuarto de fresa (Fragaria spp.) a nivel nacional (FIRA, 2016). Sin embargo, se percibe que a diferencia de los cultivos de fresa y zarzamora, la superficie cultivada de frambuesa incrementó en los últimos años, dicho incremento no puede ser explicado por cambios en el precio o rendimiento sino por otros factores. El objetivo del estudio fue determinar si hubo distorsiones en los mercados que pudieron haber influido en la elección de los productores de Jalisco por algún tipo de berry en particular. Se partió de la hipótesis, que de los berries, la frambuesa tuvo una mayor ventaja competitiva o comparativa. Para medir el grado de distorsión del mercado se aplicó la metodología de la matriz de análisis de políticas. Los resultados indican que los tres tipos de berries fueron rentables; sin embargo, algunas distorsiones económicas beneficiaron a la frambuesa y zarzamora, e impactaron negativamente al cultivo de fresa, además existió un gran subsidio a las ganancias del cultivo de frambuesa. Se concluye que distorsiones creadas por el mercado y el gobierno influyeron en la elección del tipo de berry a sembrar por los productores, favoreciendo la producción de frambuesa.

Palabras clave: decisiones de producción, distorsiones del mercado, matriz de análisis de políticas.

Recibido: septiembre de 2020

Aceptado: noviembre de 2020 


\section{Introducción}

Los frutos del bosque, también conocidos como berries (zarzamora, fresa, frambuesa y arándanos), son especies que, pese a que requieren inversiones considerables de capital para su cultivo, su elevada rentabilidad, el rápido retorno de la inversión, los altos requerimientos de mano de obra, la versatilidad en la producción de frutos para consumo y las posibilidades de exportación factibles, los convierten en cultivos con un gran potencial agrícola.

Evidencia de esto fue el crecimiento anual de $21.8 \%$ de la producción durante el periodo 20032016, en el año 2017 la producción de berries a nivel nacional fue de 390239 toneladas (SIAP, 2020) y a nivel internacional la demanda de estos también han presentado un incremento constante. México exporta aproximadamente $41 \%$ de su producción nacional de berries a Estados Unidos de América, Canadá, y los Países Bajos (SAGARPA, 2017).

Los estados en donde se ubica la mayor producción de berries son Michoacán, Jalisco, Guanajuato y Baja California (SIAP, 2020). Los berries son consumidos tradicionalmente en las regiones del norte de América y Europa; sin embargo, en los últimos años la demanda mundial de estos productos se ha incrementado, lo cual ha promovido un incremento en su producción a nivel mundial (González y Johnson, 2015).

México cuenta con un gran potencial productivo de berries, en conjunto los estados de Aguascalientes, Colima, Guanajuato, Jalisco, Michoacán, Nayarit, San Luis Potosí y Zacatecas, son capaces de dedicar cerca de 4085065 ha, a estos (SAGARPA, 2017). Este potencial productivo llevó al gobierno mexicano a impulsar a la creación y la tecnificación de dichos cultivos con técnicas como la incorporación de riego por goteo, la construcción de invernaderos y la adopción de agricultura protegida con el fin de incrementar la productividad de los berres mexicanos (Nieves et al., 2011).

Evidencia de la alta rentabilidad del cultivo es la relación beneficio costo, la cual determina la viabilidad financiera de un proyecto productivo, dicha relación es de 2.82 para el caso del blueberry, 1.88 para la frambuesa, 1.82 para la fresa y 1.76 en el caso de la zarzamora; siendo estas relaciones mayores a las observadas en cultivos básicos como la caña de azúcar y el maíz, los cuales tienen una relación beneficio costo de 1.5 y 1.2 respectivamente, esto asemeja al cultivo de berries con otros cultivos que tienen un alto índice de exportación como el aguacate, cuya relación beneficio costo es de 1.84 (González et al., 2020).

Jalisco cuenta con condiciones climáticas y edafológicas ideales para producir fresa, frambuesa y zarzamora, además de que en dicho estado se encuentran asentadas gran cantidad de empresas y asociaciones como Aneberries, Berrymex, Driscoll's dedicadas a la comercialización nacional e internacional de berries, a fin de garantizar la seguridad fitosanitaria, promover y defender el comercio y abrir nuevos mercados para este tipo de cultivos (INEGI, 2020).

Jalisco también cuenta con puertos importantes de exportación hacia China, Estados Unidos de América (EE. UU), Japón y varios países de Europa, que son los principales importadores de berries a nivel nacional (SIAVI, 2020) y de importación de los insumos necesarios para la producción, empaquetado y distribución de dichos productos. 
Si bien el estado de Jalisco cuenta con áreas dedicadas al cultivo de los tres tipos de berries, se observó que durante los últimos años existió un incremento de las áreas destinadas a la producción de frambuesa y zarzamora (Cuadro 1), mientras que las áreas destinadas a la producción de fresa disminuyeron, siendo el cultivo de frambuesa el que mostró un crecimiento más pronunciado, dicho comportamiento no puede ser explicado por su precio de venta al productor, ni por el incremento en el rendimiento provocado por la creación y adaptación de nuevas tecnologías a estos cultivos, por lo que otras distorsiones económicas podrían explicar dicho comportamiento.

Cuadro 1. Crecimiento del área sembrada, precio por tonelada, y precio de la fresa, frambuesa y zarzamora para el estado de Jalisco.

\begin{tabular}{|c|c|c|c|c|c|c|}
\hline Año & 2013 & 2014 & 2015 & 2016 & 2017 & Incremento \\
\hline \multicolumn{7}{|c|}{ Fresa } \\
\hline Área sembrada (ha) & 480 & 349 & 246 & 29 & 51 & $-841 \%$ \\
\hline Rendimiento $\left(\mathrm{t} \mathrm{ha}^{-1}\right)$ & 34 & 33 & 40 & 23 & 33 & $-3 \%$ \\
\hline Precio $\left(\$ t^{-1}\right)$ & 8893 & 9954.5 & 12065 & 14589 & 16560 & $46 \%$ \\
\hline \multicolumn{7}{|c|}{ Frambuesa } \\
\hline Área sembrada (ha) & 1512 & 1539 & 2564 & 4448 & 4482 & $66 \%$ \\
\hline Rendimiento $\left(\mathrm{t} \mathrm{ha}^{-1}\right)$ & 15 & 14 & 19 & 19 & 19 & $24 \%$ \\
\hline $\operatorname{Precio}\left(\$ \mathrm{t}^{-1}\right)$ & 14437.34 & 14771.5 & 15884.89 & 18659 & 19147 & $25 \%$ \\
\hline \multicolumn{7}{|c|}{ Zarzamora } \\
\hline Área sembrada (ha) & 431 & 364.5 & 344 & 440.9 & 536.5 & $20 \%$ \\
\hline Rendimiento $\left(\mathrm{t} \mathrm{ha}^{-1}\right)$ & 17 & 13 & 13 & 16 & 14 & $-21 \%$ \\
\hline Precio $\left(\$ t^{-1}\right)$ & 10131.5 & 8451.6 & 8772 & 11888 & 14936 & $32 \%$ \\
\hline
\end{tabular}

Elaborado con datos del Servicio de Información Agroalimentaria y Pesquera (SIAP, 2020).

La importancia de analizar cómo las políticas gubernamentales y las estructuras de mercado imperfectas afectan las decisiones de los productores, llevó a distintos autores a realizar estudios que midieran las ventajas competitivas y comparativas de distintos cultivos; a través, del uso de metodología de la matriz de análisis de políticas (MAP) cuyos resultados determinan la situación actual de la competitividad de la actividad agrícola y los instrumentos de política que la afecta (Monke y Pearson, 1989).

En 2004 se realizó un estudio para medir la evolución de la rentabilidad y competitividad del tomate rojo en Sinaloa (Hernández et al., 2004), los resultados muestran que tuvo ventajas comparativas en el mercado internacional durante el ciclo otoño-invierno 1999-2000, lo cual explicó la decisión de los productores de producir y exportar ese cultivo, en junio de 2011 se realizó un estudio similar para el cultivo de vainilla bajo distintos sistemas de producción (Barrera-Rodríguez et al., 2011).

De acuerdo a los resultados, el cultivo fue afectado negativamente por las políticas económicas como impuestos en insumos, tasas de interés y tipo de cambio sobrevaluado, reduciendo las ganancias de los productores de la región del Totonacapan, un último ejemplo es el estudio realizado con el objetivo de medir la competitividad del cultivo de trigo en Pakistán a nivel de importador y exportador (Anwar et al., 2005), en donde los autores concluyeron que el cultivo tuvo ventajas comparativas a nivel de importación, sin embargo, no presentó ninguna en exportación, lo cual limitaba su producción. 
Con base en lo anterior y para el caso de las berries se piensa que muy probablemente existieron distorsiones económicas que beneficiaron al cultivo de frambuesa en el estado de Jalisco, incrementando su rentabilidad y competitividad con respecto al cultivo de fresa y zarzamora, propiciando el crecimiento en su producción. El objetivo del estudio fue analizar y comparar la rentabilidad, competitividad y las ventajas comparativas de los cultivos de frambuesa, fresa y zarzamora de Jalisco, durante el año 2017.

La justificación de este estudio radica en la importancia económica de los tres cultivos para el estado de Jalisco, siendo este el principal productor de frambuesa, el segundo en zarzamora y el cuarto en fresa, cuya superficie sembrada en su conjunto fue de 5070 ha, con un valor de 1788 791.88 miles de pesos durante 2017 (SIAP, 2020).

\section{Materiales y métodos}

Este estudio se enfocó a los tres tipos de berries más importantes: fresa (Fragaria ananassa), frambuesa (Rubus idaeus) y zarzamora (Rubus ulmifolius), dejando de lado los arándanos azules (blueberries) y arándanos rojos debido a que estos son arbustos cuya logística de producción es distinta.

Se utilizaron los datos disponibles sobre los costos de producción de frambuesa, fresa y zarzamora, provenientes de distintas fuentes, principalmente del Sistema de Agrocostos publicado por Fideicomisos Instituidos en Relación con la Agricultura (FIRA, 2020), el Sistema Nacional de Información e Integración de Mercados (SNIIM, 2020), el Banco de México (BANXICO, 2020) y distintos manuales de siembra de berries publicados por el Instituto Nacional de Investigaciones, Forestales Agrícolas y Pecuarias (INIFAP, 2020), todos para un sistema de producción con riego por goteo, usando plántulas mejoradas y fertilización con agroquímicos para el periodo de siembra 2017 (Cuadro 2).

Cuadro 2. Estructura de los costos $(\%)$ de los cultivos de frambuesa, fresa y zarzamora.

\begin{tabular}{cccc}
\hline Ciclo & \multicolumn{3}{c}{ Perene } \\
Tecnología & Plántula mejorada - fertilizada - riego por bombeo \\
Período & \multicolumn{3}{c}{2017} \\
Superficie & Zarzamora & Fresa & Frambuesa \\
Cultivo & 42.84 & 67.58 & 59.62 \\
Insumos comercializables & 10.69 & 9.25 & 16.42 \\
Fertilizantes & 2.81 & 1.81 & 2.1 \\
Fungicidas & 0.24 & 0.15 & 0.18 \\
Herbicidas & 1.7 & 0.21 & 0.59 \\
Insecticidas & 19.33 & 48.78 & 34.02 \\
Plántulas & 7.93 & 7.29 & 6.2 \\
Otros & 0.15 & 0.09 & 0.11 \\
Diésel & 0 & 0 & 0 \\
\hline
\end{tabular}




\begin{tabular}{cccc}
\hline Factores internos & 48.15 & 28.72 & 36.85 \\
Labores manuales & 21.5 & 8.22 & 13.42 \\
Labores mecanizadas & 7.77 & 4.56 & 6.08 \\
Crédito de avío (interés) & 5.59 & 5.86 & 5.86 \\
Seguro agrícola & 4.68 & 4.89 & 4.88 \\
Uso de agua & 0.68 & 0.54 & 0.42 \\
Electricidad & 0.6 & 0.35 & 0.47 \\
Materiales diversos & 0.08 & 0.05 & 0.06 \\
Tierra & 7.25 & 4.25 & 5.67 \\
Insumos indirectamente comerciables & 0.11 & 0.06 & 0.09 \\
Tractor e implementos & 0.1 & 0.06 & 0.08 \\
Equipo de bombeo & 0.01 & 0.01 & 0.01 \\
Administración y servicios & 8.9 & 3.64 & 3.44 \\
Costo total & 100 & 100 & 100 \\
\hline
\end{tabular}

Elaborado con planes de siembra de FIRA (2020), calculando los precios y cantidades para el estado de Jalisco, precios de SNIIM (2020), paquetes tecnológicos del INIFAP (2020), tasas de interés de Banxico (2020), información de transporte de la SCT (2020) e información de productores del estado de Jalisco. Los fertilizantes incluyen reguladores de crecimiento; otros incluyen metam sodio, plástico de acolchado para la siembra y manguera de riego por goteo.

Para determinar los precios paritarios de importación, se refirió a los Estados Unidos de América como país de origen de los distintos insumos usados, en específico el estado de Oregon el cual es el principal exportador de zarzamora al territorio mexicano y California para los cultivos de fresa y frambuesa, estos precios de insumos y productos se tomaron de las bases de datos publicadas por la National Agricultural Statistics Service (USDA, 2020) y de instituciones dedicadas a la venta de insumos agrícolas para aquellos productos para los que no existe información en fuentes oficiales.

El método de análisis empleado para determinar el grado de distorsión en la rentabilidad de berries los berries fue la MAP, la cual fue desarrollada en la universidad de Cornell (Monke y Pearson, 1989), esta permite medir como las distorsiones en el mercado afectan la rentabilidad y competitividad de los productos agrícolas, así como la eficiencia en el uso de los recursos en los sistemas de producción.

La forma en que la MAP mide el impacto económico de las fallas en el mercado, es al calcular la diferencia entre los llamados precios privados que son los que se observan en la realidad, y los precios sociales que son aquellos que existirían si no ocurrieran divergencias en el mercado. La MAP está formada por tres filas, las cuales contienen el costo, ingreso y ganancia de la actividad económica calculada con los precios existentes en el mercado, llamados precios privados, la segunda con los mismos indicadores calculados con los precios que tendrían en el caso de no existir distorsiones en el mercado llamados precios económicos o precios sombra y finalmente la tercera.

Donde se miden las divergencias provocadas por el efecto de las distorsiones y políticas, en el caso de las columnas, los rendimientos se obtienen multiplicando los precios por el rendimiento por hectárea medidos en toneladas, los costos se dividen en dos, los comerciables que son aquellos que pueden adquirirse en el mercado y los factores internos que son aquellos que no se pueden comercializar entre país o bien no tienen un precio explicito como la depreciación del equipo tecnológico, finalmente la ganancia la cual es la diferencia entre los ingresos y costos (Cuadro 3). 
Cuadro 3. La matriz de análisis de política (MAP).

\begin{tabular}{ccccc}
\hline \multirow{2}{*}{ Concepto } & \multirow{2}{*}{ Ingreso } & \multicolumn{2}{c}{ Costos } & Ganancia \\
\cline { 3 - 4 } & & Bienes comerciables & Factores internos & \\
\hline Precios privados & $\mathrm{A}$ & $\mathrm{B}$ & $\mathrm{C}$ & $\mathrm{D}$ \\
Precios sociales & $\mathrm{E}$ & $\mathrm{F}$ & $\mathrm{G}$ & $\mathrm{H}$ \\
Divergencias & $\mathrm{I}$ & $\mathrm{J}$ & $\mathrm{K}$ & $\mathrm{L}$ \\
\hline
\end{tabular}

Matriz de análisis de políticas para el desarrollo agrícola (Monke y Pearson, 1989).

Donde: A y E: ingresos a precios privados y económicos B y F: costos de los bienes comerciables a precios privados y económicos $\mathrm{C}$ y $\mathrm{G}$ : costos de los factores internos a precios privados $\mathrm{y}$ económicos, $\mathrm{D}=\mathrm{A}-(\mathrm{B}+\mathrm{C})$ y $\mathrm{H}=\mathrm{E}-(\mathrm{F}+\mathrm{G})$ es la ganancia privada y económica, $\mathrm{I}=\mathrm{A}-\mathrm{E}, \mathrm{J}=\mathrm{B}-\mathrm{F}$ y $\mathrm{K}=$ $\mathrm{C}-\mathrm{G}$ miden las transferencias a través del producto, de los insumos comerciables y de los factores de producción y $\mathrm{L}=\mathrm{I}-\mathrm{J}-\mathrm{K}=\mathrm{D}-\mathrm{H}$ mide las transferencias netas.

Una vez que se recopiló la información sobre los coeficientes técnicos, los precios de insumos y productos a nivel privado y económico se crearon tres MAP's, una para cada producto analizado. Para ello se ordenó la información de manera que se pudieran identificar los bienes comerciables, que son aquellos factores de producción que pueden ser adquiridos en el mercado y por tanto tienen un precio y los factores internos, que se utilizan en la producción de estos productos pero no tienen un precio en el mercado; la información sobre el costo del riego, cantidad de jornales que se requieren para las actividades agrícolas, las actividades que se realizan con maquinaria y otros servicios contratados como asesorías técnicas, contratación de sanitarios móviles y otros.

A partir de los resultados de la MAP se pueden determinar las transferencias (subsidios e impuestos), medidas como las diferencias entre los precios privados y económicos (Cuadro 4). La magnitud de estas determina el grado de distorsión en el mercado por distintos motivos como políticas gubernamentales o existencia de estructuras de mercado imperfectas.

Cuadro 4. Matriz de análisis de política: coeficientes de protección, relaciones de eficiencia, de subsidios, de rentabilidad y de valores agregados.

\begin{tabular}{cc}
\hline Concepto & Ecuación \\
\hline Coeficiente nominal del producto (CNPP) & $\mathrm{CPNP}=\mathrm{A} / \mathrm{E}$ \\
Coeficiente nominal de insumos comerciables (CNPI) & $\mathrm{CPNI}=\mathrm{B} / \mathrm{F}$ \\
Coeficiente de protección efectiva (CPE) & $\mathrm{CPE}=(\mathrm{A}-\mathrm{B}) /(\mathrm{E}-\mathrm{F})$ \\
Eficiencia de costo privado & $\mathrm{RCP}=\mathrm{C} / \mathrm{A}-\mathrm{B})$ \\
Eficiencia del costo de los recursos internos & $\mathrm{RCR}=\mathrm{G} /(\mathrm{E}-\mathrm{F})$ \\
Subsidio social al productor & $\mathrm{SSP}=\mathrm{L} / \mathrm{E}$ \\
Equivalente al subsidio al productor & $\mathrm{ESP}=\mathrm{L} / \mathrm{A}$ \\
Subsidio a la ganancia del productor & $\mathrm{SGP}=\mathrm{D} / \mathrm{H}$ \\
Rentabilidad privada & $\mathrm{RRP}=\mathrm{D} /(\mathrm{B}+\mathrm{C})$ \\
Rentabilidad social & $\mathrm{RRE}=\mathrm{H} /(\mathrm{F}+\mathrm{G})$ \\
Valor agregado a pecios privados & $\mathrm{VAP}=(\mathrm{A}-\mathrm{B})$ \\
Valor agregado a precios económicos & $\mathrm{VAE}=(\mathrm{E}-\mathrm{F})$ \\
Consumo intermedio en el ingreso total & $\mathrm{PCIP}=(\mathrm{B} / \mathrm{A})$ \\
Valor agregado en el ingreso total & $\mathrm{PVAP}=(\mathrm{A}-\mathrm{B}) / \mathrm{A}$ \\
\hline
\end{tabular}

Matriz de análisis de políticas para el desarrollo agrícola (Monke \& Pearson, 1989). 
De los coeficientes que se obtuvieron en este estudio se analizaron aquellos que pudieran explicar el incremento en el área cultivada de frambuesa y son los siguientes: el coeficiente de protección nominal de los insumos comerciables y no comerciables, el cual mide el efecto de las distorsiones en el precio de los insumos, se obtiene de la razón entre el precio de los insumos privados.

Los cuales son los que tiene que pagar el productor a nivel de finca, entre los precios económicos de los insumos agrícolas, que son aquellos que deberían existir en caso de no existir distorsiones, si el coeficiente tiene un valor mayor a la unidad indica que el productor paga un precio mayor al que debería; es decir, que los insumos tienen un precio local mayor en relación con los mercados internacionales debido probablemente a la presencia de distorsiones en el mercado interno, lo cual es un desalentaría a los productores.

El coeficiente de protección efectiva, que mide el efecto total de las distorsiones, considerando los insumos comerciables y el producto final, se calcula dividiendo las ganancias a precios privados entre las ganancias a precios económicos. Analógicamente un valor encima de la unidad indica que el agricultor está recibiendo una ganancia mayor al que tendría en condiciones perfectas del mercado, pues en este caso la protección tanto en el producto como en los insumos genera una protección neta positiva, lo que representa ganancias para los productores.

La relación de eficiencia del costo privado $(\mathrm{RCP})$, es una relación a través de la cual se miden las ganancias creadas por la actividad agrícola y se calcula dividiendo los factores internos entre los ingresos, descontando los costos de aquellos productos que tienen un precio en el mercado, la razón de usar los precios privados es debido a que son los que están vigentes en el mercado.

Un indicador menor a la unidad expresa que se están recibiendo ganancias extraordinarias, ya que, al pagar los factores de producción, existe un residuo en el valor agregado, que es la retribución a la gestión del productor, mientras que un indicador mayor a la unidad indica que la actividad no es capaz de pagar los factores de producción y por lo tanto no se generan ganancias.

La relación de eficiencia del costo de los recursos internos (RCI), si la magnitud de este indicador se encuentra entre 0 y 1 , significa que el valor de los recursos internos (aquellos que no son adquiridos en el mercado) usados en la producción es inferior al valor de las divisas ganadas o ahorradas; esto significa que existe una ventaja comparativa en la producción interna del país analizado.

Un valor superior a la unidad indica que el valor de los recursos internos usados en la producción supera las divisas ahorradas ganas o ahorrada, por lo tanto, el país no tiene una ventaja comparativa. Una relación negativa indica que se utilizan las divisas en la producción del producto de las que este vale en el mercado. Y la relación de subsidio las cuales son: el subsidio social al productor (SSP).

El cual muestra la parte proporcional en que debería apoyarse al ingreso bruto del productor para mantener el nivel actual de ganancias privadas ante una total apertura comercial; el subsidio equivalente al productor (SEP) el cual es la divergencia total o transferencia neta de política, como una proporción de los ingresos brutos totales a precios privados y finalmente el subsidio a la ganancia del productor (SGP) el cual mide en qué grado las ganancias privadas exceden e las ganancias económicas (Lara et al., 2003). 


\section{Resultados y discusión}

Los resultados obtenidos se presentan en la Figura 1 y 2, es importante notar que debido a que el principal insecticida usado para el control de plagas en los cultivos tiene como ingrediente activo el 'paraquat' (1,1"-dimetil-4,4"-bipiridinio) y la comercialización de este agroquímico es estricta, se determinó que no existe forma de transportarse sin que haya contingencias gubernamentales que distorsionen su precio, por lo que siempre se pagara un precio más elevado por el producto.

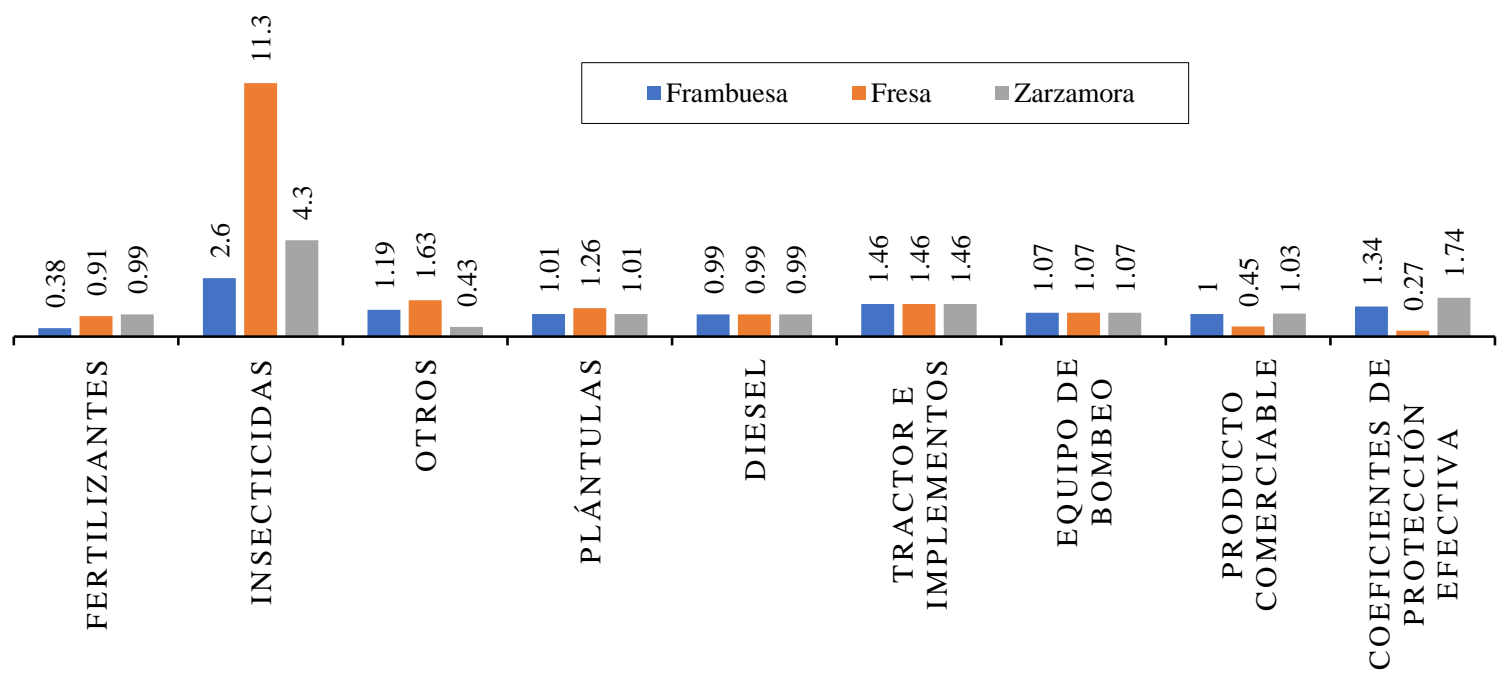

Figura 1. Coeficientes de protección nominal y efectiva para los cultivos de frambuesa, fresa y zarzamora mejorados, fertilizados y con riego por bombeo en el año 2017 para el estado de Jalisco.

Frambuesa $\quad$ Fresa $\square$ Zarzamora

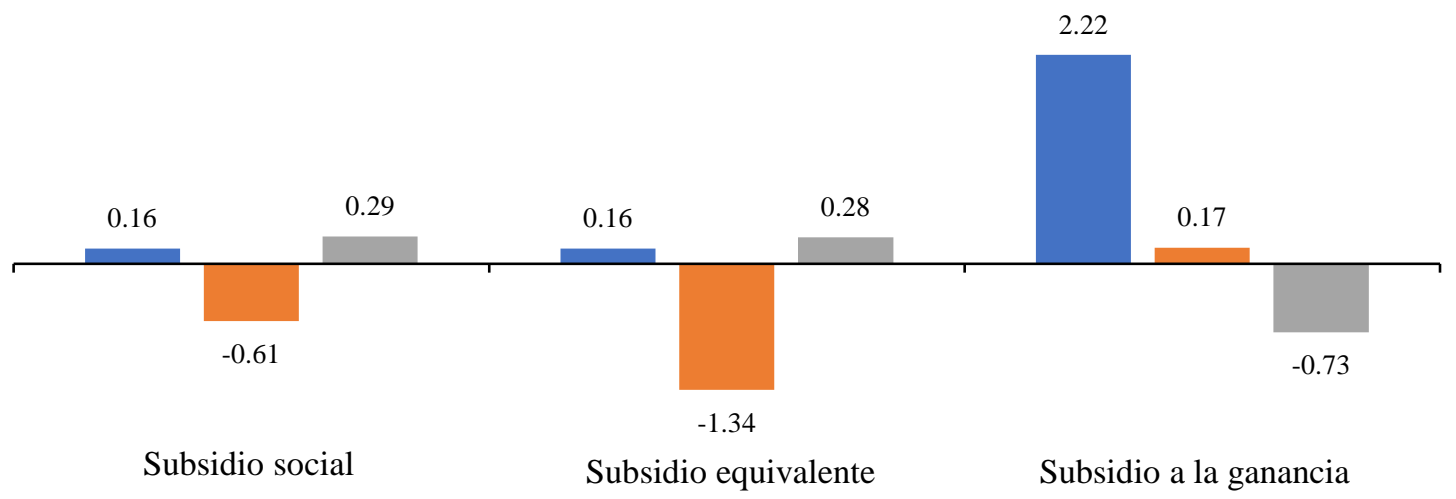

Figura 2. Relaciones de subsidios para los cultivos de frambuesa, fresa y zarzamora mejorados, fertilizados y con riego por bombeo en el año 2017 para el estado de Jalisco. 


\section{Coeficiente de protección nominal de los insumos (CNPP), coeficiente de protección nominal de los insumos (CNPI) y coeficiente de protección efectiva (CPE)}

Los indicadores de protección empleados para mostrar el grado de protección de la producción nacional con respecto a la producción extranjera son el CNPP y el CPE, el primero ayuda a entender la manera en que las políticas de precios incentiva o desincentiva a los productores, mientras que el segundo es una medida del grado de transferencia a producto e insumos derivados de la política comercial y del tipo de cambio.

Los resultados muestran que las políticas beneficiaron a los productores en la adquisición de fertilizantes, en especial para el caso de la frambuesa, los cuales adquirieron estos productos por $38 \%$ del precio que debieron haber pagado, y el diésel agrícola que para todos los cultivos se pagó un precio menor. Sin embargo, se observa que en todos los casos existió una desprotección en la adquisición de insecticidas, plántulas e implementos mecánicos, para el caso de los insecticidas los productores pagaron más del doble, los de zarzamora el cuádruple y los de fresa presentaron la mayor desprotección teniendo que pagar once veces más de lo necesario; para el caso de los implementos, todos tuvieron que pagar $50 \%$ más por la adquisición de tractores e implementos agrícolas.

Al analizar y comparar el subsidio al producto comerciable podemos observar que tanto la frambuesa como zarzamora no presentaron un grado de protección o desprotección importante; sin embargo, para el caso de la fresa el producto presentó un alto nivel de desprotección, ya que el precio obtenido fue aproximadamente $50 \%$ menor al que debió tener.

Como medida de protección total tenemos que, al comparar el coeficiente de protección efectiva, la frambuesa y zarzamora presentaron un grado de protección importante ya que el valor obtenido es mayor a la unidad, siendo el segundo el más beneficiado con un valor de 1.74, mientras que la fresa presentó un grado muy alto de desprotección (Figura 1). Los resultados demuestran que a pesar de que los tres cultivos fueron rentables, la fresa presentó un alto grado de desprotección, desincentivando a los productores y guiándolos a elegir los cultivos de frambuesa y zarzamora.

\section{Relaciones de eficiencia del costo privado (RCP) y del costo de los factores internos (RCI)}

La relación de costo privado (RCP) permitió comparar la eficiencia privada, los resultados mostraron que en los tres cultivos las RCP resultaron con valores positivos mayores a cero, por lo que los cultivos son rentables y competitivos, para la frambuesa el indicador fue de 0.49 , la fresa 0.47 y la zarzamora 0.8 , de tal manera que resulta más conveniente producirlo internamente que importarlo, siendo la frambuesa el cultivo más competitivo, y la zarzamora el menos competitivo, dado que su valor es el más cercano a la unidad.

Es importante resaltar, que aunque estos valores fueron mayores a los observados en otros cultivos de exportación como el aguacate, el cual en promedio tiene indicadores cercanos a 0.1 , lo cual implica una menor competitividad, los berries tienen una mayor relación beneficio costo, aunque la producción de aguacate resulte más competitiva (Franco et al., 2018). 
Comparativamente un estudio realizado con la finalidad de medir la rentabilidad de la producción de maíz en el estado de Guanajuato (Guzmán et al., 2014) obtuvo como resultados 0.04 para la producción de maíz en temporal, 0.13 en riego asistido con ganado y 0.85 en riego asistido sin ganado; lo cual significa que un importante factor a considerar al analizar la rentabilidad de un producto, es el grado de desarrollo tecnológico presente a nivel de finca (González y Alferes, 2010), factor que está presente en un alto grado en los cultivos de berries, en especial en el caso de la frambuesa debido al manejo postcosecha que este producto requiere.

Para la relación de eficiencia de los costos de los recursos internos (RCI), los valores fueron de 0.69 para frambuesa, 0.12 para fresa y 1.46 para zarzamora, en este caso la fresa y frambuesa presentan una ventaja comparativa en relación con la producción de USA, ya que por cada peso invertido 0.69 y 0.12 son para el pago de factores internos y el restante es posible utilidad.

\section{Relaciones de subsidio social al productor (SSP), subsidio equivalente al productor (SEP) y subsidio a la ganancia del productor (SGP)}

$\mathrm{Al}$ analizar las relaciones de subsidio y enfocarnos particularmente en la relación a la ganancia del productor (SGP), observamos que la frambuesa presentó un importante subsidio a las ganancias, las ganancias privadas excedieron a las económicas en más del doble, esto significa que existe un gran incentivo para producir este producto por encima de los otros, de acuerdo a los resultados obtenidos la frambuesa obtuvo un valor de 2.22 , la fresa 0.17 , y la zarzamora -0.73 , esto significa que las ganancias privadas están sobrevaluadas para el caso de la frambuesa, lo cual coloca a la primera por encima de otros cultivos para el comercio internacional del estado, como el tomate rojo el cual tiene un valor de 0.54 (Hernández et al., 2004), mientras que lo contrario ocurre para el caso de la fresa donde las ganancias están subvaluadas $83 \%$ con respecto a las económica.

En el caso de los otros dos indicadores podemos observar que la frambuesa también resultó más beneficiada en comparación con el cultivo de zarzamora (para la fresa por tener valor negativo no existe interpretación ya que uno de los valores resulta negativo), pues el valor del subsidio al productor es menor, por lo que está menos expuesto ante una apertura total, y la divergencia de las transferencias como proporción de los ingresos brutos es menor (Figura 2).

\section{Conclusiones}

La Matriz de Análisis de Política (MAP) permitió identificar y cuantificar algunas de las distorsiones de las políticas sobre los precios que existen en los sistemas de producción de los berries estudiados en la presente investigación (fresa, frambuesa y zarzamora) lo cual permitió estimar el costo que representa para los productores destinar sus recursos para la producción de un tipo de berrie determinado, de tal manera que la MAP brinda los elementos para visualizar la rentabilidad tanto desde el punto de vista del productor y como aquella tomando en cuenta el uso eficiente de los recursos productivos del país.

Los resultados señalan que la razón del incremento en la superficie sembrada de frambuesa en el año 2017, se debió en gran medida a la protección que existieron en el mercado de berries, las cuales beneficiaron en mayor proporción al cultivo frambuesa. Hubo un mayor margen de ganancias para los productores ya que los subsidios en la adquisición de fertilizantes y el beneficio en el precio de la frambuesa resultaron mucho mayores a los impuestos en los demás insumos. 
La frambuesa también presentó una mayor competitividad con respecto a la frambuesa producida en Estados Unidos incrementado su potencial de exportación. En cambio, dichas distorsiones y fallas perjudicaron a los productores de fresa y zarzamora (en especial a los primeros) desincentivando su producción. Si la protección a la frambuesa continúa, es de prever que la superficie dedicada este cultivo siga en aumento mientras que las de fresa y zarzamora probablemente se mantendrán estables o incluso disminuyan.

De acuerdo con algunos estudios, una de las razones por las que el cultivo de zarzamora ha dejado de producirse en muchas de las regiones de Jalisco ha sido la alta incidencia de plagas y enfermedades, las cuales se han incrementado durante los últimos años. Los resultados de este trabajo respaldan dicha aseveración, se puede observar que existe una desprotección en la adquisición insecticidas para los productores de zarzamora, los cuales deben pagar un precio más elevado para adquirir dichos agroquímicos, resultado en la adquisición y aplicación de plaguicidas en dosis que no son óptimas, provocando la proliferación de plagas y enfermedades.

Los resultados obtenidos en este artículo coinciden con los de otros estudios realizados para analizar el mercado de berries en México, más allá de las condiciones geográficas, existen distintas ventajas las cuales han impulsado la producción de las berries, factores como la existencia de una fuerte base institucional y gubernamental, el desarrollo y la adaptación de nuevas técnicas, y la inserción de México en la cadena global han impulsado de adopción en la producción de estos cultivos; sin embargo, la interferencia gubernamental y la existencia de agentes que controlan los precios de insumos y productos, representan una amenaza para el desarrollo y expansión en la producción en Jalisco de los berries.

\section{Literatura citada}

Anwar, S.; Hussain, Z. and S. J. M. 2005. Comparative advantage and competitiveness of wheat crop in Pakistan. Lahore Journal of Economics, Department of Economics, The Lahore School of Economics. USDA. National Agricultural Statics Service. United States Department of Agriculture. 10(2):101-110. https://www.nass.usda.gov/.

Banxico. 2020. Banco de México. Indicadores económicos. https://www.banxico.org.mx/.

Barrera-Rodríguez, A. I.; Jaramillo-Villanueva, J. L.; Escobedo-Garrido, J. S. y Herrera-Cabrera, B. E. 2011. Rentabilidad y competitividad de los sistemas de producción de vainilla (Vanilla planifolia J.) en la región del Totonacapan, México. Agrociencia. 45(5):625-638.

FIRA. 2015. Fideicomisos Instituidos en Relación a la Agricultura. Panorama Agroalimentario. Berries 2016.https://www.gob.mx/cms/uploads/attachment/file/200633/panorama_ agroalimentario_berries_2016.pdf.

FIRA. 2020. Fideicomisos Instituidos en Relación con la Agricultura. Agrocostos. https://www.fira.gob.mx/nd/agrocostos.jsp.

Franco, S. M. A.; Leos, R. J. A.; Salas, G. J. M.; Acosta, R. M. and García, M. A. 2018. Analysis of costs and competitiveness in avocado production in Michoacán, Mexico. Rev. Mex. Cienc. Agríc. 9(2):391-404.

González, A. and Alferes, M. 2010. Competitiveness and comparative advantage of maize production in Mexico. Rev. Mex. Cienc. Agríc. 1(3):381-396. 
González, M. E. and Johnson, M. H. 2015. Anthocyanins From berries: chemistry and roles in inflammation and diabetes. Encyclopedia of Life Support Systems (EOLSS)/UNESCO, 15. Singapure.

González, R. M. G.; Santoyo, C. V. H.; Arana, C. J. J. and Muñoz, R. M. 2020. The insertion of Mexico into the global value chain of berries. World Development Perspectives. 20:100240. https://doi.org/10.1016/j.wdp.2020.100240.

Guzmán, S. E.; De La Garza, C. M. T.; García, S. J. A.; Rebollar, R. S. y Hernández, M. J. 2014. Rentabilidad privada de la producción de maíz en la región Bajío de Guanajuato, México. Rev. Facultad Nacional de Agronomía Medellín. 67(2):7291-7299.

Hernández, M. J.; García, M. R.; Vaca, A.; Valdivia, A. R. y Omaña, S. J. M. 2004. Evolución de la competitividad y rentabilidad del cultivo del tomate rojo (Lycopersicon esculentum L.) en Sinaloa, México. Agrociencia. 38(4):431-436.

INEGI. 2020. Instituto Nacional de Estadística y Geografía. Directorio de empresas y establecimientos. https://inegi.org.mx/temas/directorio/.

INIFAP. 2020. Instituto Nacional de Investigación Forestal y Agropecuaria. https://www.gob.mx/inifap.

Lara, C. D.; Mora, F. J. S.; Martínez, D. M. A.; García, D. G.; Omaña, S. J. M. y Gallegos, S. J. 2003. Competitividad y ventajas comparativas de los sistemas producción de leche en el estado de Jalisco, México. Agrociencia. 37(1):85-94.

Monke, E. A. and Pearson, S. R. 1989. The policy analysis matrix for agricultural development. Cornell University Press. Ithaca, New York., USA and London England. 279 p.

Nieves, G. V.; Van-der, V. O. and Elings, A. 2011. Mexican protected horticulture: production and market of Mexican protected horticulture described and analysed. Wageningen UR Greenhouse Horticulture/LEI. 104 p. https://edepot.wur.nl/196070.

SAGARPA. 2017. Secretaría de Agricultura, Ganadería, Desarrollo Rural, Pesca y Agricultura. Planeación Agrícola Nacional 2017-2030. Frutas del bosque. https://www.gob.mx/ cms/uploads/attachment/file/257076/potencial-frutas_del_bosque.pdf.

SCT. 2020. Secretaría de Comunicaciones y Transportes. Rutas punto a punto. Traza tu ruta. http://app.sct.gob.mx/sibuac_internet/controllerui?action=cmdescogeruta.

SIAP. 2017. Servicio de Información Agroalimentaria y Pesquera. Datos anuales de producción y valor de producción de fresa, frambuesa y zarzamora. https://www.gob.mx/siap.

SIAVI. 2020. Sistema de información arancelaria Vía Internet. Secretaría de Economía. http://www.economia-snci.gob.mx/.

SNIIM. 2020. Sistema Nacional de Información e Integración de Mercados. Secretaría de Economía. http://www.economia-sniim.gob.mx/nuevo/.

USDA. 2020. National Agricultural Statics Service. United States Department of Agriculture: https://www.nass.usda.gov/. 